\title{
Residual Stress Measurements Inside a Small Inner Diameter Hole at Low Bragg Angle Using X-Ray Diffraction Technique
}

\author{
M. Belassel ${ }^{1, a^{*}}$, J. Pineault ${ }^{1, b}, M$. Brauss $^{2, c}$. \\ ${ }^{1}$ Proto Manufacturing Limited, 2175 Solar Crescent, Oldcastle, Ontario, Canada \\ ${ }^{2}$ Proto Manufacturing Inc., 12350 Universal Drive, Taylor, Michigan, 48180, USA

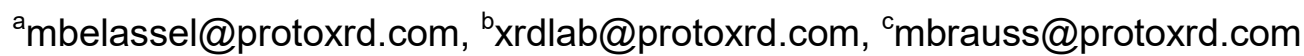

Keywords: Residual Stress, Bolt-Hole, Inner Diameter, Low Bragg Angle, Abusive Machining

\begin{abstract}
A new X-Ray diffraction (XRD) goniometer has been specially developed to nondestructively measure residual stress (RS) on the inner diameter (ID) of small holes in metal and ceramic components. The major advantage of this novel goniometer is its ability to perform RS measurements on the ID of small holes without the need to section the component, thus maintaining the integrity of the residual stress field inherent to the component of interest. This new patented XRD goniometer has been used on a wide variety of aerospace components on features such as holes in airframe/structural components, as well as fastener/bolt holes, air holes, and confined areas on rotating and non-rotating turbine engine components. In many instances, measurements can be performed at $2 \theta$ angles much lower than the widely accepted lower limit of approximately $130^{\circ}$. The selection of the actual Bragg angle used depends upon the material itself, the hole ID, and the thickness of the component. Results obtained indicate that the new goniometer can be used to measure RS on the ID of small holes with good accuracy and precision even at relatively low $2 \theta$ angles in the vicinity of $100^{\circ}$.
\end{abstract}

\section{Introduction}

Since the discovery of RS and its measurement early in the last century, measurement techniques have been evolving steadily [1]. Many techniques have been developed thanks to industry demand and specific needs [2]. Many RS measurement techniques exist and each generally requires a different experimental setup; laboratory equipment is often suitable for small parts, whereas portable systems are generally required for oversized parts. Moreover, the geometry of the XRD goniometer may vary to facilitate access to the measurement locations of interest on a component. Both Psi and Omega modes have been widely implemented on most current XRD RS measurement instruments. Omega mode is suitable for tight geometries such as grooves as may be found in the root of a gear tooth when measuring along the groove (i.e. parallel to the teeth), whereas Psi mode in turn is more adapted to measurements in the direction normal (perpendicular) to the groove (such as the opening of the groove permits). Each geometry has its advantages and disadvantages and each may be complementary in certain instances.

In this study, RS analysis will be extended to measurements on the ID of bolt holes in the hoop direction. Such measurements are applicable for both automotive and aerospace materials; this study includes examples on a Ni-Base super-alloy. For these applications, a new XRD instrument and goniometer were designed and fabricated that operate in Psi mode [3]. 


\section{Stress Measurement Technique}

Basic Principles. The x-ray diffraction technique uses the distance between crystallographic planes, i.e. the d-spacing, as a strain gage in a crystalline material. When the material is in tension, the dspacing increases and when the material is in compression, the d-spacing decreases. The presence of residual stresses in a material produces a shift in the x-ray diffraction peak angular position that is directly measured by the detector [3]. The diffraction angle $2 \theta$ is measured experimentally and the $\mathrm{d}$ spacing is then calculated using Bragg's law:

$$
n \lambda=2 d \sin \theta
$$

Where: $\lambda$ is the wavelength of the radiation.

Diffraction peaks collected should have a sufficient peak/background ratio and a limited noise level. These will depend on the $\{h k l\}$ plane selected, the collection time, the size of the irradiated area and the material microstructure. Once the d-spacing is measured for unstressed $d_{0}$ and stressed $d$ conditions, the strain is calculated using either of the following relationships:

$$
\varepsilon=\frac{\left(d-d_{0}\right)}{d_{0}} \quad \text { or } \quad \varepsilon=-\frac{1}{2} \cot (\theta) \cdot \Delta(2 \theta)
$$

To improve measurement accuracy when determining $\Delta(2 \theta)$, the $\theta$ angle selected should be close to $90^{\circ}$ (i.e. $2 \theta \sim 180^{\circ}$ ). The widely published and agreed upon low limit for $2 \theta$ is $130^{\circ}$; using $2 \theta$ values below this will diminish $\Delta(2 \theta)$ resolution and as such will result in a loss in RS measurement accuracy and a concomitant increase in experimental error. The plot in Figure 1 demonstrates the relationship between $\Delta(2 \theta)$ for various $2 \theta$ values. As $2 \theta$ deceases so does the slope, indicating a reduced resolution in peak separation.

$\operatorname{Sin}^{2} \psi$ Method. For the $\sin ^{2} \psi$ method, where a number of d-spacings are measured, stresses are calculated from an equation derived from Hooke's law for isotropic, homogeneous, fine grain materials, $\psi$ is the angle subtended by the bisector of the incident and diffracted beam and the surface normal, and $\phi$ is the

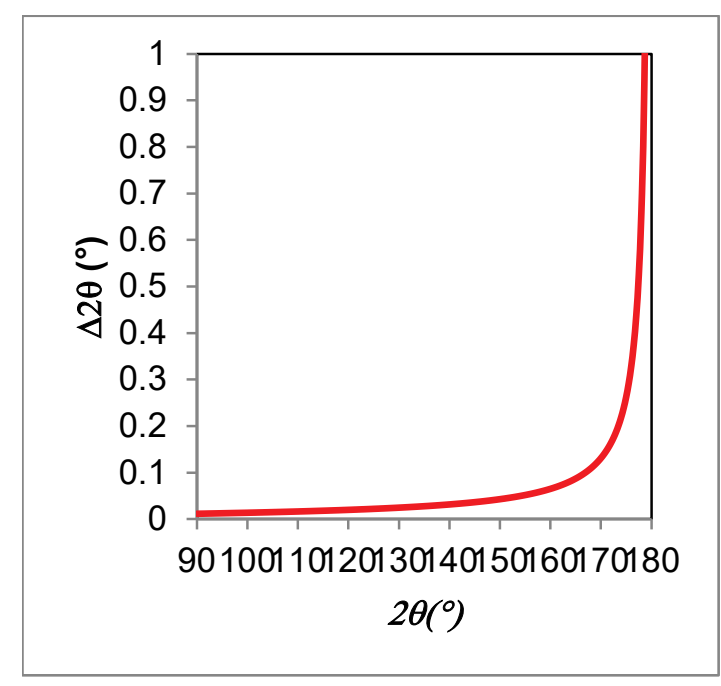

Figure 1: Variation in $\Delta(2 \theta)$ resolution with diffraction angle $2 \theta$. direction of stress measurement [4].

$$
\frac{d_{\phi \psi}-d_{0}}{d_{0}}=\frac{1}{2} S_{2}{ }^{h k l}\left(\sigma_{\phi}-\sigma_{33}\right) \sin ^{2} \psi+1 / 2 S_{2}{ }^{h k l} \sigma_{33}-S^{h k l}{ }_{1}\left(\sigma_{11}+\sigma_{22}+\sigma_{33}\right)+\frac{1}{2} S_{2}{ }^{h k l} \tau_{\phi} \sin 2 \psi
$$

Where: $\varepsilon_{\phi \psi}$ is the strain at a given $\psi, \phi$ tilts, $1 / 2 \mathrm{~S}_{2}{ }^{\mathrm{hkl}}$ and $\mathrm{S}_{1}{ }^{\mathrm{hkl}}$ are the x-ray elastic constants of the material. 


\section{Materials and Measurement Conditions}

The material of interest for this study is a Ni-base super-alloy. Three examples of surface conditions that can be found on the ID of machined holes were investigated as follows:

1. Hole drilled with no coolant.

2. Hole drilled with coolant.

3. Drilled, honed and shot peened hole.

Residual stress versus depth profiles were collected on the face of each of the machined holes using conventional XRD instrumentation and on the ID of the same holes using a new XRD instrument. The measurement conditions were as follows: 1) for the conventional instrument operating in Omega mode, the $\{311\}$ diffraction plane was selected for measurements at $2 \theta=151.9^{\circ}$ using $\mathrm{Mn}_{\mathrm{k \alpha}}$ radiation ( $\lambda=2.1034$ Angstroms), and 2) for the new instrument operating in Psi mode, the $\{311\}$ diffraction plane was selected for measurements at $2 \theta=111.3^{\circ}$ using $\mathrm{Co}_{\mathrm{k} \alpha}$ radiation $(\lambda=1.7902$ Angstroms). Selection of the diffraction angle $2 \theta$ to be employed using the new instrument is predicated upon the diameter of the hole $(D)$, and the thickness of the component $(W)$. A geometric relationship can thus be established as seen in Eq. 4 and in Figure 2:

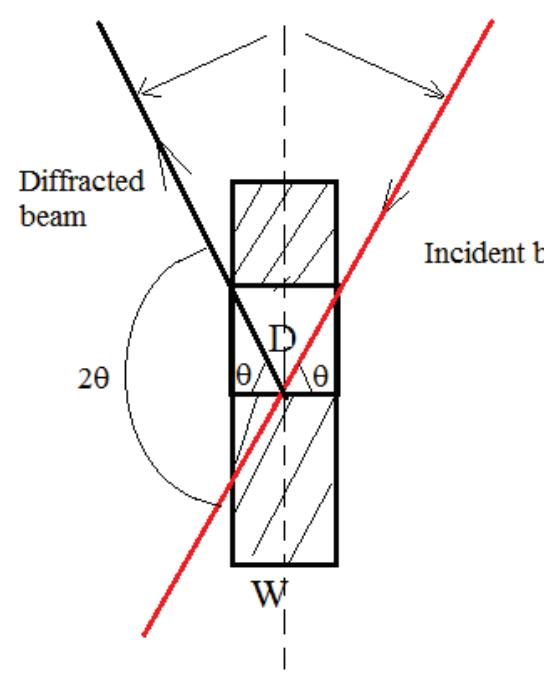

Figure 2: Determining optimal $2 \theta$ for use on the ID of a hole using new Psi mode instrument.

$$
\tan \left(\theta_{\max }\right)=\frac{2 D}{W}
$$

For Ni-base super-alloys where the actual collimator and the detector size are taken into account, the optimal $2 \theta$ to use is $111.3^{\circ}$. In this case, the ratio is: $\frac{2 D}{W}=4,2 \theta_{\max }=152^{\circ}$, which is widely sufficient for this experiment. For other materials, the ratio may vary according to the Bragg conditions available for use and the component geometry.

In Figure 3, the RS on a Ni-base super-alloy turbine engine disk is being measured using: a) a conventional Omega mode instrument on the face of the hole, and b) using the new instrument in Psi mode on the ID of the same hole. 

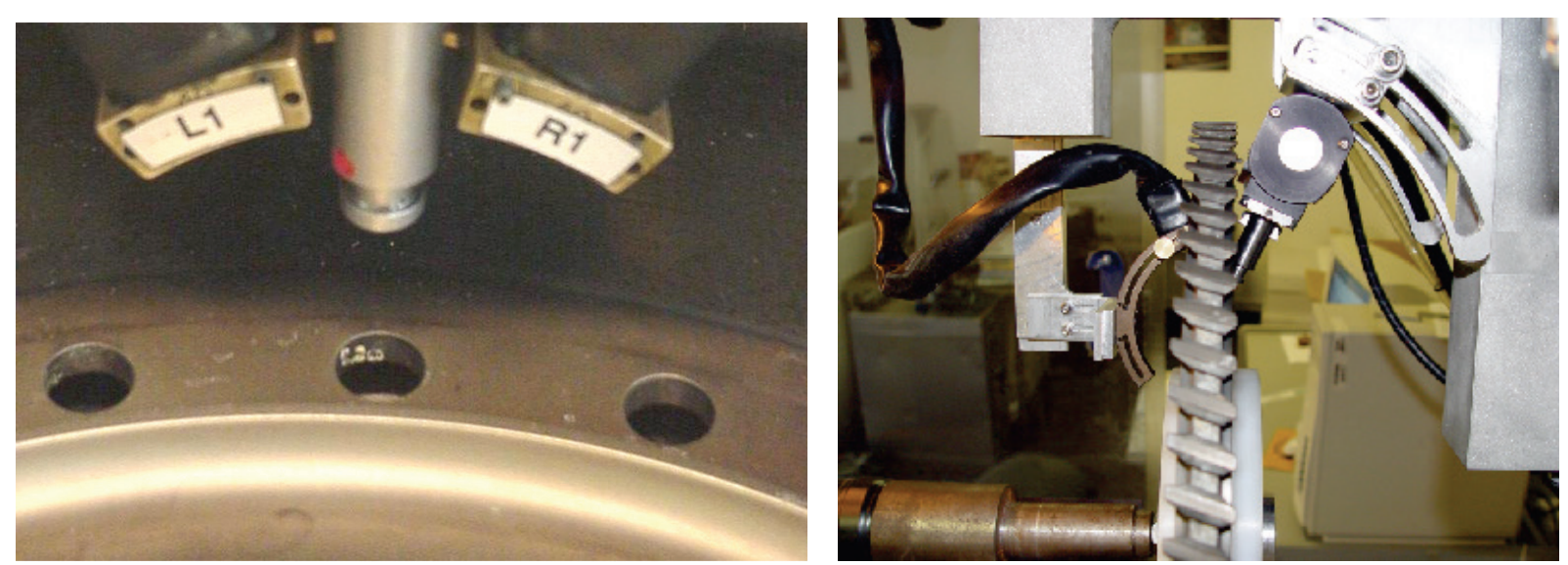

Figure 3: Measuring RS a) on the face of machined holes using a conventional XRD instrument and $b)$ on the ID of machined holes using the new XRD instrument

\section{Results and Discussion}

Prior to performing RS measurements, the alignment of the instrument was verified using a stress free Ni powder as per ASTM E915 [5], as well as a known high stress proficiency standard.

Microstructure characterization of the different processes revealed the following:

1. The hole drilled with no coolant exhibited severely distorted grains and undesirable microstructure near the hole ID surface.

2. The hole drilled with coolant exhibited only slightly distorted grains near the hole ID surface.

3. The drilled, honed and shot peened hole showed no signs of abusively machined material.

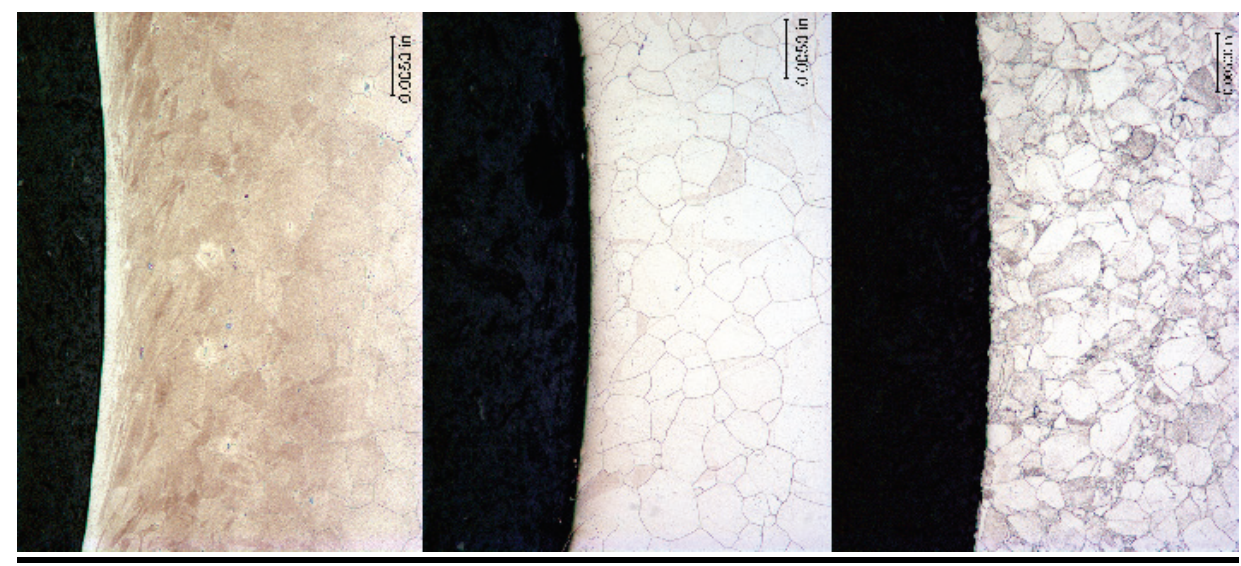

(a)

(b)

(c)

Figure 4 - Hole cross sections (ID to the left) of holes machined as follows: a) drilled with no coolant, b) drilled with coolant, and c) drilled, honed and shot peened. 
The RS vs. depth results obtained on both the face of the hole, and the ID of the hole, can be seen in Figure 5. The results obtained on the ID of the machined holes were found to be much more effective for detecting the relatively thin tensile stressed layers inherent to the various machining and cold working processes applied. In the case of the hole drilled with coolant, RS that appeared compressive on the face of the disk, was found to be tensile on the hole ID. In the case of the hole drilled without coolant, the magnitude of the tensile RS found on the ID was much greater when compared to tensile RS found on the face of the disk. Thus, the very steep stress gradients produced by machining demand nondestructive measurement of RS on the ID.
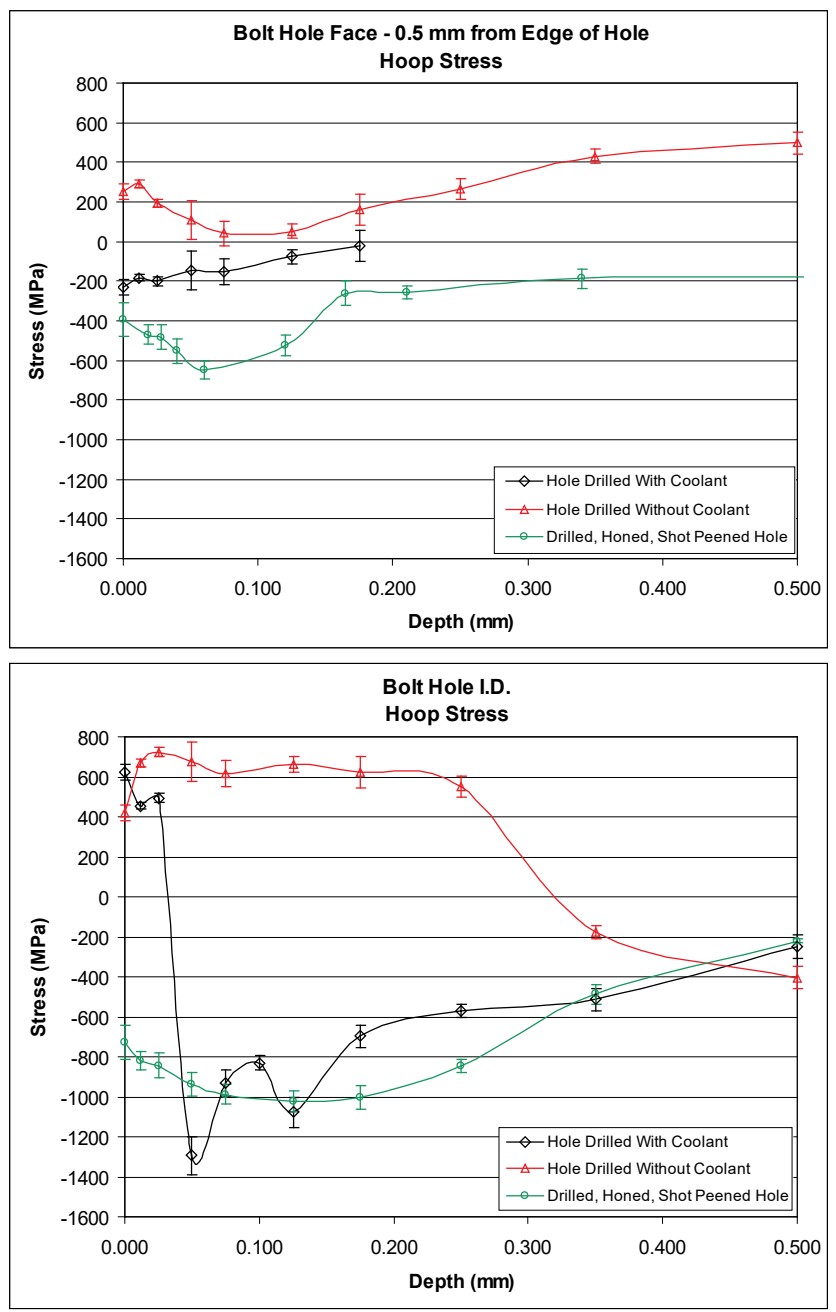

a)

b)

Figure 5: Stress vs. depth profiles a) on the face of machined holes using a conventional XRD instrument, and b) on the ID of machined holes using a new XRD instrument.

Clearly, the new XRD instrument adapted for measurements on the ID surface was more effective in revealing the actual stress gradients present i.e., it was more effective in revealing the effect of each machining and cold working processes applied as well as the associated depth, sign and magnitude of undesirable tensile RS [6]. This ability is critical for the aerospace industry where accurate RS measurements are needed to help improve and optimize the machining and cold working processes applied to life limiting components so as to insure a long service life of such components. 
The experimental errors associated with the $2 \theta$ angles selected should be higher for low $2 \theta$ and vice versa [1]. The magnitude of the experimental errors obtained in this study was deemed good given the magnitude of the RS present and the associated RS gradients observed [7]. The magnitude of the experimental errors were found to be dependent upon the RS levels measured, as well as the material condition and microstructural effects present in the hole that were investigated. A detailed methodology to successfully measure RS at a suitable Bragg angle (20) was developed prior to applying it to real components. This methodology also employs a statistical approach where each measurement is reproduced to guarantee its accuracy.

\section{Summary}

New goniometer head geometry has been developed and successfully applied to a new instrument that can be used to measure RS on the ID of small holes nondestructively. Such measurements were heretofore impossible to execute without sectioning the component. The application of this technique on real components presented many challenges that were addressed using a new, high accuracy instrument (and associated methodology) to reliably measure RS on the ID of small holes. Moreover, the new instrument provided RS results that correctly characterized the actual RS state present on the disk hole ID where a conventional instrument failed. This new instrument thus opens the way to the accurate evaluation of RS on the ID of small holes which is of great interest to both industry in general, and to the aerospace industry in particular.

\section{References}

[1] V. Hauk, "Structural and Residual Stress Analysis by Nondestructive Methods", Elsevier, Amsterdam, 1997.

[2] J.A. Pineault, M. Belassel, M.E. Brauss, "X-Ray Diffraction Residual Stress Measurement in Failure Analysis", Failure Analysis and Prevention, Vol. 11, ASM Metals Handbook, American Society for Metals, 2002, p. 484-497.

[3] M.Brauss, Proto Patent No. US 7,283,612 B2, issued October 16, 2007 for X-Ray Diffraction Method.

[4] Noyan I.C. and Cohen J.B., 'Residual Stress', Springer Verlag, 1987.

http://dx.doi.org/10.1007/978-1-4613-9570-6

[5] ASTM E915-10, "Standard test method for verifying the alignment of x-ray diffraction instrumentation for residual stress measurement".

[6] M. Belassel et al., "Effect of Thermal and Mechanical Loading on the Generation of Macro and Micro Stresses in Eutectoid Steel", Proc. 4th Int. Conf. Residual Stresses, ICRS 4. Soc. Exp. Mechanics, 1994, 392-401.

[7] J. Lu et al. "Handbook of Measurement of Residual Stresses", SEM, Published by the Fairmont Press, Inc., 1996. 